\title{
The Generalized Reed-Muller codes in a modular group algebra
}

\author{
Harinaivo ANDRIATAHINY \\ Mention: Mathématiques et informatique, \\ Domaine: Sciences et Technologies, \\ Université d'Antananarivo, Madagascar \\ e-mail: hariandriatahiny@gmail.com \\ Vololona Harinoro RAKOTOMALALA \\ Mention: Météorologie, \\ Domaine: Sciences de l'Ingénieur, \\ Ecole Supérieure Polytechnique d'Antananarivo, \\ Université d'Antananarivo, Madagascar \\ e-mail: volhrakoto@gmail.com
}

October 28, 2018

\begin{abstract}
First we study some properties of the modular group algebra $\mathbb{F}_{p^{r}}[G]$ where $G$ is the additive group of a Galois ring of characteristic $p^{r}$ and $\mathbb{F}_{p^{r}}$ is the field of $p^{r}$ elements. Secondly a description of the Generalized Reed-Muller codes over $\mathbb{F}_{p^{r}}$ in $\mathbb{F}_{p^{r}}[G]$ is presented.
\end{abstract}

Keywords: Generalized Reed-Muller codes, group algebra, Galois ring, Jennings basis.

MSC 2010: 94B05, 94B15, 20C05, 16N40, $12 \mathrm{E} 05$.

\section{Introduction}

It is well known that the Reed-Muller codes of length $p^{m}$ over the prime field $\mathbb{F}_{p}$ are the radical powers of the modular group algebra $\mathbb{F}_{p}\left[\mathbb{F}_{p^{m}}\right]([3],[4])$.

In general, this property is no longer true for the Generalized Reed-Muller (GRM) codes over a non prime field $\mathbb{F}_{p^{r}}(r>1)$. Let $M$ be the radical of the modular group algebra $\mathbb{F}_{p^{r}}[G]$ where $G$ is the additive group of the Galois ring $G R\left(p^{r}, m\right)$ of characteristic $p^{r}$ and of rank $m$. Since $\mathbb{F}_{p^{r}}[G]$ is isomorphic to $\mathbb{F}_{p^{r}}\left[X_{0}, X_{1}, \ldots, X_{m-1}\right] /\left(X_{0}^{p^{r}}-1, \ldots, X_{m-1}^{p^{r}}-1\right)$ (see Proposition 5.1), then as shown in [1], except for $M^{0}, M$ and $M^{m\left(p^{r}-1\right)}$, none of the radical powers of 
$\mathbb{F}_{p^{r}}[G]$ is a GRM code over the non prime field $\mathbb{F}_{p^{r}}$.

Many authors have described the GRM codes in group algebras, especially in the group algebra $\mathbb{F}_{p^{r}}[H]$ where $H$ is an elementary abelian p-group or $H$ is the additive group of an extension field of $\mathbb{F}_{p^{r}}([4],[5],[9])$.

We give a description of the GRM codes over an arbitrary finite field $\mathbb{F}_{p^{r}}$ in the group algebra $\mathbb{F}_{p^{r}}[G]$ where $G$ is the additive group of the Galois ring $G R\left(p^{r}, m\right)$. We organize this paper as follows. In section 2, we recall the definition and some properties of the Galois ring $G R\left(p^{r}, m\right)$. In section 3, we summarize some results on the group algebra $\mathbb{F}_{p^{r}}\left[\mathbb{F}_{p^{m}}\right]$ developed by Assmus and Key in [2]. In particular, some facts about a linear basis of the group algebra $\mathbb{F}_{p^{r}}\left[\mathbb{F}_{p^{m}}\right]$ called Jennings basis ([7]) are pointed out. In section 4, We give some generalizations of the properties of $\mathbb{F}_{p^{r}}\left[\mathbb{F}_{p^{m}}\right]$ presented in section 3 by considering the group algebra $\mathbb{F}_{p^{r}}[G]$ where $G$ is the additive group of the Galois ring $G R\left(p^{r}, m\right)$. In section 5 , we establish a natural isomorphism between $\mathbb{F}_{p^{r}}[G]$ and $\mathbb{F}_{p^{r}}\left[X_{0}, X_{1}, \ldots, X_{m-1}\right] /\left(X_{0}^{p^{r}}-1, \ldots, X_{m-1}^{p^{r}}-1\right)$. In section 6 , we describe the GRM codes over a finite field $\mathbb{F}_{p^{r}}$ in $\mathbb{F}_{p^{r}}[G]$ by using the fact that GRM codes are extended cyclic codes. In section 7 , an example is given.

\section{Galois ring}

Let us start with some basic properties of Galois ring (see [10]).

Set $\mathbb{Z}_{p^{r}}:=\mathbb{Z} / p^{r} \mathbb{Z}$ and let $h(X) \in \mathbb{Z}_{p^{r}}[X]$ be a monic basic irreducible polynomial of degree $m$. Such a polynomial always exists by Hensel's Lemma. The Galois ring of characteristic $p^{r}$ and of rank $m$, denoted by $G R\left(p^{r}, m\right)$, is defined as the quotient ring

$$
\mathbb{Z}_{p^{r}}[X] /(h(X)) .
$$

In particular, $G R\left(p^{r}, 1\right)=\mathbb{Z}_{p^{r}}$ and $G R\left(p^{1}, m\right)=\mathbb{F}_{p^{m}}$.

$G R\left(p^{r}, m\right)$ is a local ring with maximal ideal $p G R\left(p^{r}, m\right)$ and residue field

$$
G R\left(p^{r}, m\right) / p G R\left(p^{r}, m\right) \cong \mathbb{F}_{p^{m}} .
$$

The Galois ring $G R\left(p^{r}, m\right)$ is a finite chain ring; all the ideals of $G R\left(p^{r}, m\right)$ are ordered as follows:

$$
\{0\}=p^{r} G R\left(p^{r}, m\right) \subset p^{r-1} G R\left(p^{r}, m\right) \subset \ldots \subset p G R\left(p^{r}, m\right) \subset G R\left(p^{r}, m\right) .
$$

Each element $v \in G R\left(p^{r}, m\right)$ can be expressed as $v=p^{t} u$ where $u$ is an invertible element in $G R\left(p^{r}, m\right)$ and in this expression $t$ is unique up to modulo $p^{r-t}$. Finally, we recall that if $m_{1}$ is a positive integer such that $m_{1} \mid m$, then $G R\left(p^{r}, m_{1}\right)$ is a subring of $G R\left(p^{r}, m\right)$. Conversely, any subring of $G R\left(p^{r}, m\right)$ is of the form $G R\left(p^{r}, m_{1}\right)$ for some $m_{1} \mid m$.

\section{$3 \quad$ The group algebra $\mathbb{F}_{p^{r}}\left[\mathbb{F}_{p^{m}}\right]$}

We recall in this section some properties of the group algebra $\mathbb{F}_{p^{r}}\left[\mathbb{F}_{p^{m}}\right]$ treated in [2]. Let $F:=\mathbb{F}_{p^{r}}$ be any subfield of $\mathbb{F}_{p^{m}}$. Set $G$ equal to the additive group 
of the finite field $\mathbb{F}_{p^{m}}$. Thus $G$ can be viewed as an elementary abelian $p$-group of order $p^{m}$. Set

$$
R=F[G]
$$

the group algebra of $G$ over the field $F$. Using polynomial notation, an element of $R$, which is a function from $G$ to $F$, is a formal sum $\sum_{g \in G} a_{g} X^{g}$ where the coefficient $a_{g}$ is simply the value of the function at the group element $g$. The operations in the group algebra $R$ are defined as follows:

$$
\begin{gathered}
\sum_{g \in G} a_{g} X^{g}+\sum_{g \in G} b_{g} X^{g}=\sum_{g \in G}\left(a_{g}+b_{g}\right) X^{g} \\
\left(\sum_{g \in G} a_{g} X^{g}\right)\left(\sum_{g \in G} b_{g} X^{g}\right)=\sum_{g, h \in G} a_{g} b_{h} X^{g+h}=\sum_{k \in G}\left(\sum_{h \in G} a_{k-h} b_{h}\right) X^{k}
\end{gathered}
$$

and, for $c \in F$,

$$
c\left(\sum_{g \in G} a_{g} X^{g}\right)=\sum_{g \in G}\left(c a_{g}\right) X^{g} .
$$

$X^{0}$ is the multiplicative identity of the group algebra $R$; i.e $X^{0} a=a$ for every $a \in R$. We will simply denote it by 1 .

The augmentation map

$$
\begin{aligned}
\phi: \quad R & \longrightarrow F \\
\sum_{g \in G} a_{g} X^{g} & \longmapsto \sum_{g \in G} a_{g}
\end{aligned}
$$

is an algebra homomorphism.

The kernel of $\phi$ is denoted by

$$
M=\left\{\sum_{g \in G} a_{g} X^{g} \in R \mid \sum_{g \in G} a_{g}=0\right\} .
$$

Thus, $M$ is an ideal of $R$.

Since $G$ is an elementary abelian $p$-group, we have

$$
\left(\sum_{g \in G} a_{g} X^{g}\right)^{p}=\sum_{g \in G}\left(a_{g}\right)^{p} X^{p g}=\sum_{g \in G}\left(a_{g}\right)^{p} X^{0}=\left(\sum_{g \in G} a_{g}\right)^{p} X^{0} .
$$

3.1 Proposition. $R$ is a local group algebra with unique maximal ideal $M=\operatorname{Rad}(R)=\left\{a \in R \mid a^{p}=0\right\}$.

3.2 Remark. Let $p$ be a prime number. Then

1. $(-1)^{p-1}=1 \bmod p$.

2. $\left(\begin{array}{c}p-1 \\ i\end{array}\right)=(-1)^{i} \bmod p$ for all $i=0,1, \ldots, p-1$. 
3.3 Proposition. Provided $g \neq 0$, we have

$$
\left(X^{g}-1\right)^{p-1}=\sum_{i=0}^{p-1} X^{i g}
$$

3.4 Remark. Let $U$ be a non-empty subset of $G$. Then

$$
\prod_{g \in U}\left(X^{g}-1\right)^{p-1}= \begin{cases}\sum_{g \in\langle U\rangle} X^{g} & \text { if } U \text { is a linearly independent set } \\ 0 & \text { otherwise }\end{cases}
$$

where $\langle U\rangle$ is the subspace spanned by $U$.

Let $S$ be a subset of $R=F[G]$. The annihilator of $S$ is defined by

$$
\operatorname{Ann}(S):=\{a \in R \mid a s=0 \text { for all } s \in S\} .
$$

3.5 Theorem. Let $G$ be an elementary abelian p-group of order $p^{m}, F$ a field of characteristic $p$ and $R=F[G]$.

1. For any basis $\left\{g_{0}, \ldots, g_{m-1}\right\}$ of $G$, the $p^{m}$ elements $\prod_{\nu=0}^{m-1}\left(X^{g_{\nu}}-1\right)^{e_{\nu}}$ where $0 \leq e_{\nu}<p$ form a linear basis for $R$.

2. Moreover, $\left\{\prod_{\nu=0}^{m-1}\left(X^{g_{\nu}}-1\right)^{e_{\nu}} \mid \sum_{\nu=0}^{m-1} e_{\nu} \geq t, 0 \leq e_{\nu}<p\right\}$ form a linear basis of $M^{t}$, where $M$ is the radical of $R$.

3. $\operatorname{Ann}\left(M^{t}\right)=m^{m(p-1)+1-t}$.

3.6 Definition. The basis for $F[G]$ given in theorem 3.5. is called a Jennings basis of the group algebra.

\section{The group algebra $\mathbb{F}_{p^{r}}\left[G R\left(p^{r}, m\right)\right]$}

In this section, we give some natural generalizations of the properties of the group algebra $\mathbb{F}_{p^{r}}\left[\mathbb{F}_{p^{m}}\right]$ formulated in section 3 .

Let $F$ be the field $\mathbb{F}_{p^{r}}$ of $p^{r}$ elements where $p$ is a prime number and $r \geq 1$ is an integer. Set $G$ equal to the additive group of the Galois ring $G R\left(p^{r}, m\right)$. Set

$$
R=F[G]=\left\{\sum_{g \in G} a_{g} X^{g} \mid a_{g} \in F\right\}
$$

the group algebra of $G$ over the field $F$. The operations in $R$ are defined as in section 3 .

We consider the ideal $M:=\left\{\sum_{g \in G} a_{g} X^{g} \in R \mid \sum_{g \in G} a_{g}=0\right\}$ of $R$.

Since $G$ is of characteristic $p^{r}$, we have

$$
\left(\sum_{g \in G} a_{g} X^{g}\right)^{p^{r}}=\sum_{g \in G}\left(a_{g}\right)^{p^{r}} X^{p^{r} g}=\sum_{g \in G} a_{g} X^{0}=\left(\sum_{g \in G} a_{g}\right) X^{0} .
$$


4.1 Proposition. $R$ is a local group algebra with unique maximal ideal

$$
M=\operatorname{Rad}(R)=\left\{a \in R \mid a^{p^{r}}=0\right\} .
$$

Proof. The relation (6) shows that $\left(\sum_{g \in G} a_{g} X^{g}\right)^{p^{r}}$ is zero if and only if $\sum_{g \in G} a_{g}=0$. Thus, $M$ is the radical of $R$.

Moreover, it shows also that every element of $R$ that is not in $M$ is a unit of $R$ and it follows that $M$ is the unique maximal ideal of $R$.

4.2 Remark. The set $\left\{X^{g} \mid g \in G\right\}$ is a linear basis of $R$ considered as a vector space over $F$.

4.3 Proposition. The ideal $M$ is generated linearly over the field $F$ by the elements $X^{g}-1$ where $g \in G-\{0\}$.

Proof. Each element $X^{g}-1$ is in $M$. Conversely, let $\sum_{g \in G} a_{g} X^{g} \in M$, i.e $\sum_{g \in G} a_{g}=0$. We have

$$
\begin{aligned}
\sum_{g \in G} a_{g} X^{g} & =\sum_{g \in G} a_{g}\left[\left(X^{g}-1\right)+1\right] \\
& =\sum_{g \in G}\left[a_{g}\left(X^{g}-1\right)+a_{g} 1\right]=\sum_{g \in G} a_{g}\left(X^{g}-1\right)+\sum_{g \in G} a_{g} 1 \\
& =\sum_{g \in G} a_{g}\left(X^{g}-1\right)+\left(\sum_{g \in G} a_{g}\right) 1=\sum_{g \in G} a_{g}\left(X^{g}-1\right) \\
& =\sum_{g \in G-\{0\}} a_{g}\left(X^{g}-1\right) .
\end{aligned}
$$

4.4 Lemma. Let $p$ be a prime number and $r \geq 1$ an integer. Then

1. $(-1)^{p^{r}-1}=1 \bmod p$.

2. $\left(\begin{array}{c}p^{r}-1 \\ i\end{array}\right)=(-1)^{i} \bmod p$ for all $i=0,1, \ldots, p^{r}-1$.

Proof. The first assertion is trivial. The second can be proved by induction on $i$.

4.5 Proposition. Provided $g \neq 0$, we have

$$
\left(X^{g}-1\right)^{p^{r}-1}=\sum_{i=0}^{p^{r}-1} X^{i g}
$$

Proof.

$$
\begin{aligned}
\left(X^{g}-1\right)^{p^{r}-1} & =\sum_{i=0}^{p^{r}-1}\left(\begin{array}{c}
p^{r}-1 \\
i
\end{array}\right)(-1)^{p^{r}-1-i} X^{i g} \\
& =\sum_{i=0}^{p^{r}-1}\left(\begin{array}{c}
p^{r}-1 \\
i
\end{array}\right)(-1)^{p^{r}-1}(-1)^{-i} X^{i g}=\sum_{i=0}^{p^{r}-1} X^{i g}
\end{aligned}
$$


by Lemma 4.4 .

From (11), notice that

$$
\begin{aligned}
G R\left(p^{r}, m\right) & =\mathbb{Z}_{p^{r}}[X] /(h(X)) \\
& =\left\{i_{0}+i_{1} \alpha+\ldots+i_{m-1} \alpha^{m-1} \mid i_{l} \in \mathbb{Z}_{p^{r}}, 0 \leq l \leq m-1\right\}
\end{aligned}
$$

where $\alpha:=X+(h(X))$.

4.6 Proposition. We have

$$
\left(X^{1}-1\right)^{p^{r}-1}\left(X^{\alpha}-1\right)^{p^{r}-1} \ldots\left(X^{\alpha^{m-1}}-1\right)^{p^{r}-1}=\sum_{g \in G} X^{g}
$$

Proof. By Proposition 4.5, we have

$$
\begin{aligned}
& \left(X^{1}-1\right)^{p^{r}-1}\left(X^{\alpha}-1\right)^{p^{r}-1} \ldots\left(X^{\alpha^{m-1}}-1\right)^{p^{r}-1} \\
& =\left(\sum_{i_{0}=0}^{p^{r}-1} X^{i_{0} 1}\right)\left(\sum_{i_{1}=0}^{p^{r}-1} X^{i_{1} \alpha}\right) \ldots\left(\sum_{i_{m-1}=0}^{p^{r}-1} X^{i_{m-1} \alpha^{m-1}}\right) \\
& =\sum_{0 \leq i_{l} \leq p^{r}-1,0 \leq l \leq m-1} X^{i_{0} 1} X^{i_{1} \alpha} \ldots X^{i_{m-1} \alpha^{m-1}} \\
& =\sum_{0 \leq i_{l} \leq p^{r}-1,0 \leq l \leq m-1} X^{i_{0} 1+i_{1} \alpha+\ldots+i_{m-1} \alpha^{m-1}}=\sum_{g \in G} X^{g}
\end{aligned}
$$

Set

$$
E:=\left\{0,1, \ldots, p^{m r}-1\right\}
$$

the set of integers between 0 and $p^{m r}-1$. Let $i$ be an element of $E$ and

$$
i=i_{0}+i_{1} p^{r}+i_{2} p^{2 r}+\ldots+i_{m-1} p^{(m-1) r}
$$

where $0 \leq i_{l} \leq p^{r}-1$ for all $l=0,1, \ldots, m-1$ its $p^{r}$-adic expansion. The $p^{r}$-weight of $i$ is defined by

$$
\omega_{p^{r}}(i):=\sum_{l=0}^{m-1} i_{l} .
$$

Then, set

$$
V_{i}:=\left(X^{1}-1\right)^{i_{0}}\left(X^{\alpha}-1\right)^{i_{1}} \ldots\left(X^{\alpha^{m-1}}-1\right)^{i_{m-1}}
$$

and

$$
B_{t}:=\left\{V_{i} \mid i \in E, \omega_{p^{r}}(i) \geq t\right\}
$$

for all $t$ such that $0 \leq t \leq m\left(p^{r}-1\right)$.

4.7 Proposition. $B_{0}$ is a linear basis of $R$ over $F$. 
Proof. We have $\operatorname{card}\left(B_{0}\right)=p^{r m}=\operatorname{dim}_{F}(R)$.

Let us prove that $B_{0}$ is a linearly independant set over $F$. Consider the linear combination $\sum_{\omega_{p r}(i) \geq 0} \lambda_{i} V_{i}=0$, where $\lambda_{i} \in F$ for all $i$. We have $\lambda_{0} V_{0}+$ $\sum_{\omega_{p r}(i) \geq 1} \lambda_{i} V_{i}=0$ where $V_{0}=1$ is the identity element of $R$. Multiplying by $V_{p^{r m}-1}$, we get $\lambda_{0} V_{p^{r m}-1}+\sum_{\omega_{p^{r}}(i) \geq 1} \lambda_{i} V_{i} V_{p^{r m}-1}=0$.

Let $i=\sum_{l=0}^{m-1} i_{l} p^{l r}$ such that $\omega_{p^{r}}(i) \geq 1$. Then, there is an integer $l(0 \leq l \leq m-$ $1)$ such that $i_{l} \geq 1$. thus, $i_{l}+p^{r}-1 \geq p^{r}$. Since $\left(X^{g}-1\right)^{p^{r}}=0$ for any $g$, we have $V_{i} V_{p^{r m}-1}=\left(X^{1}-1\right)^{i_{0}+p^{r}-1} \ldots\left(X^{\alpha^{l}}-1\right)^{i_{l}+p^{r}-1} \ldots\left(X^{\alpha^{m-1}}-1\right)^{i_{m-1}+p^{r}-1}=0$. Then, $0=\lambda_{0} V_{p^{r m}-1}=\lambda_{0} \sum_{g \in G} X^{g}$ by Proposition 4.6. Hence, $\lambda_{0}=0$.

Thus, we have $\sum_{\omega_{p r}(i) \geq 1} \lambda_{i} V_{i}=0$.

Let $i=\sum_{l=0}^{m-1} i_{l} p^{l r}$ such that $\omega_{p^{r}}(i)=1$.

We have $\lambda_{i} V_{i}+\sum_{\omega_{p^{r}}(j) \geq 1, j \neq i} \lambda_{j} V_{j}=0$. Multiplying by $V_{p^{r m}-1-i}$, we have $\lambda_{i} V_{i} V_{p^{r m}-1-i}+\sum_{\omega_{p r}(j) \geq 1, j \neq i} \lambda_{j} V_{j} V_{p^{r m}-1-i}=0$.

Let $j=\sum_{l=0}^{m-1} j_{l} p^{l r} \neq i$ such that $\omega_{p^{r}}(j) \geq 1=\omega_{p^{r}}(i)$. Then, there is an integer $l(0 \leq l \leq m-1)$ such that $j_{l}>i_{l}$, and hence $j_{l} \geq i_{l}+1$ and $j_{l}+p^{r}-1-i_{l} \geq$ $\left(i_{l}+1\right)+p^{r}-1-i_{l}=p^{r}$. Since $\left(X^{g}-1\right)^{p^{r}}=0$ for any $g$, we have $V_{j} V_{p^{r m}-1-i}=$ $\left(X^{1}-1\right)^{j_{0}+p^{r}-1-i_{0}} \ldots\left(X^{\alpha^{l}}-1\right)^{j_{l}+p^{r}-1-i_{l}} \ldots\left(X^{\alpha^{m-1}}-1\right)^{j_{m-1}+p^{r}-1-i_{m-1}}=0$. Consequently, $0=\lambda_{i} V_{i} V_{p^{r m}-1-i}=\lambda_{i} \sum_{g \in G} X^{g}$.

Hence $\lambda_{i}=0$.

Continuing in this way, we have

$\lambda_{i}=0$ for all $i$ such that $\omega_{p^{r}}(i)=1$, and

$\lambda_{i}=0$ for all $i$ such that $\omega_{p^{r}}(i)=2,3, \ldots, m\left(p^{r}-1\right)$.

4.8 Corollary. $B_{1}$ is a linear basis of $M$ over $F$.

Proof. Since $B_{1} \subset B_{0}$, then $B_{1}$ is a linearly independant set of $R$ over $F$. And since $V_{i} \in M$ for all $i$ such that $\omega_{p^{r}}(i) \geq 1, B_{1} \subseteq M$. Moreover, we have $\operatorname{dim}_{F}(M)=p^{r m}-1=\operatorname{card}\left(B_{1}\right)$.

4.9 Corollary. $B_{t}$ is a linear basis of $M^{t}$ over $F$ where $1 \leq t \leq m\left(p^{r}-1\right)$.

Proof. Since $B_{t} \subset B_{0}, B_{t}$ is a linearly independant set of $R$ over F. $M^{t}$ is generated as ideal by the set $\left\{a_{1} a_{2} \ldots a_{t} \mid a_{i} \in M, 1 \leq i \leq t\right\}$. Consider a product $a_{1} a_{2} \ldots a_{t}$ where $a_{i} \in M$ for all $i(1 \leq i \leq t)$. Since $M$ is linearly generated by $B_{1}$ over $F$, then we have $a_{i}=\sum_{j=1}^{p^{r m}}-1 \lambda_{i_{j}} V_{j}$, where $\lambda_{i_{j}} \in F$ for all $i$ and $j$. Therefore, $a_{1} a_{2} \ldots a_{t}=\prod_{i=1}^{t}\left(\sum_{j=1}^{p^{r m}}-1 \lambda_{i_{j}} V_{j}\right)=\sum_{\omega_{p^{r}}(j) \geq t} \beta_{j} V_{j} \in B_{t}$ where $\beta_{j} \in F$. And it is clear that a finite sum of such products is a linear combination of the elements of $B_{t}$ with coefficients in $F$. Thus, $M^{t}$ is generated by $B_{t}$ over $F$.

4.10 Remark. The index of nilpotency of the radical $M$ is $1+m\left(p^{r}-1\right)$. And we have the following sequence of ideals:

$$
\{0\}=M^{1+m\left(p^{r}-1\right)} \subset M^{m\left(p^{r}-1\right)} \subset \ldots \subset M^{2} \subset M \subset R .
$$

4.11 Proposition. $\operatorname{Ann}\left(M^{t}\right)=M^{1+m\left(p^{r}-1\right)-t}$ where $1 \leq t \leq m\left(p^{r}-1\right)$. 
Proof. Let $t$ be an integer such that $1 \leq t \leq m\left(p^{r}-1\right)$. By Corollary 4.9] (10), (9) and (7), $B_{t}:=\left\{V_{i} \mid i \in E, \omega_{p^{r}}(i) \geq t\right\}$ is a linear basis of $M^{t}$ over $F$ where $V_{i}:=\left(X^{1}-1\right)^{i_{0}}\left(X^{\alpha}-1\right)^{i_{1}} \ldots\left(X^{\alpha^{m-1}}-1\right)^{i_{m-1}}$ and $i=i_{0}+i_{1} p^{r}+\ldots+$ $i_{m-1} p^{(m-1) r}\left(0 \leq i_{l} \leq p^{r}-1, \forall l\right)$.

By definition, $\operatorname{Ann}\left(M^{t}\right):=\left\{a \in R \mid a s=0\right.$ for all $\left.s \in M^{t}\right\}$.

Since the index of nilpotency of $M$ is $1+m\left(p^{r}-1\right)$, then we have $\operatorname{Ann}\left(M^{t}\right) \supseteq$ $M^{1+m\left(p^{r}-1\right)-t}$.

Let $a \in \operatorname{Ann}\left(M^{t}\right)$. Since $a \in R$, we have $a=\sum_{\omega_{p^{r}}(i) \geq 0} \lambda_{i} V_{i}$ where $\lambda_{i} \in F$ for all $i$.

Let $i$ such that $\omega_{p^{r}}(i)=m\left(p^{r}-1\right)$. Thus, $i=p^{r m}-1$.

Since $V_{p^{r m}-1} \in M^{m\left(p^{r}-1\right)} \subseteq M^{t}$, then $a V_{p^{r m}-1}=\lambda_{0} V_{p^{r m}-1}=\lambda_{0} \sum_{g \in G} X^{g}=0$.

Hence, $\lambda_{0}=0$.

Let $i \in E$ such that $\omega_{p^{r}}(i)=m\left(p^{r}-1\right)-1$.

Since $V_{i} \in M^{m\left(p^{r}-1\right)-1} \subseteq M^{t}$, then $a V_{i}=\lambda_{\bar{i}} \sum_{g \in G} X^{g}=0$ where $\bar{i}=\left(p^{r}-\right.$ $\left.1-i_{0}\right)+\left(p^{r}-1-i_{1}\right) p^{r}+\ldots+\left(p^{r}-1-i_{m-1}\right) p^{(m-1) r}$. Hence $\lambda_{\bar{i}}=0$. We have $\omega_{p^{r}}(\bar{i})=m\left(p^{r}-1\right)-\omega_{p^{r}}(i)=1$. Therefore, $\lambda_{\bar{i}}=0$ for all $i$ such that $\omega_{p^{r}}(i)=m\left(p^{r}-1\right)-1$.

Let $i$ such that $\omega_{p^{r}}(i)=m\left(p^{r}-1\right)-2$.

Since $V_{i} \in M^{m\left(p^{r}-1\right)-2} \subseteq M^{t}$, then $a V_{i}=\lambda_{\bar{i}} \sum_{g \in G} X^{g}=0$. Hence $\lambda_{\bar{i}}=0$. It follows that $\lambda_{\bar{i}}=0$ for all $i$ such that $\omega_{p^{r}}(i)=m\left(p^{r}-1\right)-2$, i.e $\omega_{p^{r}}(\bar{i})=2$. Continuing in this way, finally let $i$ such that $\omega_{p^{r}}(i)=t$.

Since $V_{i} \in M^{t}$, then $a V_{i}=\lambda_{\bar{i}} \sum_{g \in G} X^{g}=0$. Hence $\lambda_{\bar{i}}=0$ with $\omega_{p^{r}}(\bar{i})=$ $m\left(p^{r}-1\right)-t$.

It follows that $\lambda_{\bar{i}}=0$ for all $i$ such that $\omega_{p^{r}}(i)=t$, i.e $\omega_{p^{r}}(\bar{i})=m\left(p^{r}-1\right)-t$.

Then $a \in M^{m\left(p^{r}-1\right)-t+1}$.

\section{$5 \quad$ Isomorphism between $\mathbb{F}_{p^{r}}\left[G R\left(p^{r}, m\right)\right]$ and $\mathbb{F}_{p^{r}}\left[X_{0}, X_{1}, \ldots, X_{m-1}\right] /\left(X_{0}^{p^{r}}-1, \ldots, X_{m-1}^{p^{r}}-1\right)$}

Recall that $\mathbb{F}_{p^{r}}[G]=\left\{\sum_{g \in G} a_{g} X^{g} \mid a_{g} \in \mathbb{F}_{p^{r}}\right\}$ where $G$ is the additive group of the Galois ring $G R\left(p^{r}, m\right)$.

5.1 Proposition. The group algebra $\mathbb{F}_{p^{r}}[G]$ is isomorphic to the quotient ring $\mathbb{F}_{p^{r}}\left[X_{0}, X_{1}, \ldots, X_{m-1}\right] /\left(X_{0}^{p^{r}}-1, \ldots, X_{m-1}^{p^{r}}-1\right)$.

Proof. Consider the mapping

$$
\begin{aligned}
\psi: \quad \mathbb{F}_{p^{r}}\left[X_{0}, X_{1}, \ldots, X_{m-1}\right] & \longrightarrow R \\
X_{i} & \longmapsto X^{\alpha^{i}}
\end{aligned}
$$

where $\alpha$ is defined in (7).

It is clear that the ideal $\left\langle X_{i}^{p^{r}}-1 \mid 0 \leq i \leq m-1\right\rangle \subseteq \operatorname{ker} \psi$.

Conversely, let $a\left(X_{0}, \ldots, X_{m-1}\right) \in \operatorname{ker} \psi$. By using the division algorithm in $\mathbb{F}_{p^{r}}\left[X_{0}, X_{1}, \ldots, X_{m-1}\right]\left(\right.$ see[6]) with the lexicographic order such that $X_{0}>$ 
$X_{1}>\ldots>X_{m-1}$, let us divide $a\left(X_{0}, \ldots, X_{m-1}\right)$ by

$\left\{X_{0}^{p^{r}}-1, \ldots, X_{m-1}^{p^{r}}-1\right\}$. We have

$a\left(X_{0}, \ldots, X_{m-1}\right)=b_{0}\left(X_{0}^{p^{r}}-1\right)+\ldots+b_{m-1}\left(X_{m-1}^{p^{r}}-1\right)+g\left(X_{0}, \ldots, X_{m-1}\right)$

where $b_{i} \in \mathbb{F}_{p^{r}}\left[X_{0}, X_{1}, \ldots, X_{m-1}\right]$ for all $i$ and

$g\left(X_{0}, \ldots, X_{m-1}\right)=\sum_{0 \leq i_{k} \leq p^{r}-1} g_{i_{0}, \ldots, i_{m-1}} X_{0}^{i_{0}} \ldots X_{m-1}^{i_{m-1}}$

with $g_{i_{0}, \ldots, i_{m-1}} \in \mathbb{F}_{p^{r}}$.

Then

$$
\begin{aligned}
\psi\left(a\left(X_{0}, \ldots, X_{m-1}\right)\right) & =\psi\left(g\left(X_{0}, \ldots, X_{m-1}\right)\right) \\
& =\sum_{0 \leq i_{k} \leq p^{r}-1} g_{i_{0}, \ldots, i_{m-1}} X^{i_{0}+i_{1} \alpha+\ldots+i_{m-1} \alpha^{m-1}}=0 .
\end{aligned}
$$

By Remark 4.2, $g_{i_{0}, \ldots, i_{m-1}}=0$ for all $i_{k}$ such that $0 \leq i_{k} \leq p^{r}-1(0 \leq k \leq$ $m-1)$. This implies that $g\left(X_{0}, \ldots, X_{m-1}\right)=0$ and thus $a\left(X_{0}, \ldots, X_{m-1}\right) \in$ $\left\langle X_{i}^{p^{r}}-1 \mid 0 \leq i \leq m-1\right\rangle$.

\section{Description of the GRM codes in $\mathbb{F}_{p^{r}}\left[G R\left(p^{r}, m\right)\right]$}

Set $n:=q^{m}-1$ where $q=p^{r}, p$ is a prime number and $r, m$ are integers $\geq 1$. Let $A=\mathbb{F}_{q}[Z] /\left(Z^{n}-1\right)$. We have $A=\left\{a_{0}+a_{1} z+\ldots+a_{n-1} z^{n-1} \mid a_{i} \in \mathbb{F}_{q}\right\}$ where $z=Z+\left(Z^{n}-1\right)$.

The shortened Generalized Reed-Muller code of length $n$ and of order $\nu(0 \leq$ $\nu<m(q-1))$ over $\mathbb{F}_{q}$ denoted by $H C_{\nu}(m, q)$ is the ideal of $A$ generated by

$$
f_{\nu}(z)=\prod_{0<\omega_{q}(i) \leq m(q-1)-\nu-1}\left(z-\gamma^{i}\right)
$$

where $\gamma$ is a primitive element of the finite field $\mathbb{F}_{q^{m}}([8],[11])$, and $\omega_{q}(i)$ is defined in (8).

Recall that $G$ is the additive group of the Galois ring $G R\left(p^{r}, m\right)$. Let us order the elements of $G=\left\{0, g_{0}, g_{1}, \ldots, g_{q^{m}-2}\right\}$ as follows:

$$
0<g_{0}<g_{1}<\ldots<g_{q^{m}-2} .
$$

Thus, we have the following identification:

$$
\sum_{g \in G} a_{g} X^{g} \in \mathbb{F}_{q}[G] \longleftrightarrow\left(a_{g}\right)_{g \in G} \in\left(\mathbb{F}_{q}\right)^{q^{m}} .
$$

Let us order the monomials $\left\{X^{g} \mid g \in G\right\}$ such that $X^{g_{i}}<X^{g_{j}}$ if and only if $g_{i}<g_{j}$.

For $a=\sum_{g \in G} a_{g} X^{g} \in \mathbb{F}_{q}[G]$, set $\operatorname{md}(a):=\max \left\{g \in G \mid a_{g} \neq 0\right\}$.

We define the leading term of $a$ by $\operatorname{LT}(a):=a_{\operatorname{md}(a)} X^{\operatorname{md}(a)}$.

Consider the linear transformation

$$
\begin{aligned}
\varphi: A & \longrightarrow \mathbb{F}_{q}[G] \\
a_{0}+a_{1} z+\ldots+a_{n-1} z^{n-1} & \longmapsto\left(-\sum_{i=0}^{n-1} a_{i}\right) X^{0}+\sum_{i=0}^{n-1} a_{i} X^{g_{i}}
\end{aligned}
$$


The Generalized Reed-Muller codes of length $q^{m}$ and of order $\nu(0 \leq \nu<$ $m(q-1))$ over $\mathbb{F}_{q}$ is defined by

$$
C_{\nu}(m, q)=\varphi\left(H C_{\nu}(m, q)\right) .
$$

We have the following sequence:

$$
\{0\} \subset C_{0}(m, q) \subset C_{1}(m, q) \subset \cdots \subset C_{m(q-1)-1}(m, q) \subset \mathbb{F}_{q}[G]
$$

Since $\varphi$ is a one to one morphism, then we have

$$
\operatorname{dim}_{\mathbb{F}_{q}}\left(C_{\nu}(m, q)\right)=\operatorname{dim}_{\mathbb{F}_{q}}\left(H C_{\nu}(m, q)\right)
$$

with

$$
\operatorname{dim}_{\mathbb{F}_{q}}\left(H C_{\nu}(m, q)\right)=q^{m}-1-\operatorname{deg}\left(f_{\nu}(z)\right) .
$$

Set

$$
\theta_{\nu}^{j}=\varphi\left(z^{j} f_{\nu}(z)\right)
$$

where $0 \leq \nu \leq m(q-1)-1$.

By (13), we have

$$
\operatorname{LT}\left(\theta_{\nu}^{j}\right)=X^{g_{\operatorname{deg}\left(z^{j} f_{\nu}(z)\right)}} .
$$

Set $N(t):=\operatorname{card}\left(\left\{k \in\left[0, q^{m}-1\right] \mid \omega_{q}(k)=t\right\}\right)$ where $\left[0, q^{m}-1\right]$ is the set of integers between 0 and $q^{m}-1$.

6.1 Theorem. We have

1. $C_{0}(m, q)$ is linearly generated over $\mathbb{F}_{q}$ by $\theta_{0}^{0}$.

2. $C_{\nu}(m, q)$ is linearly generated over $\mathbb{F}_{q}$ by the set

$$
\begin{aligned}
& K_{\nu}:=\left\{\theta_{0}^{0}\right\} \cup\left\{\theta_{i}^{j} \mid 1 \leq i \leq \nu, 0 \leq j \leq N(m(q-1)-i)-1\right\} \text { where } 1 \leq \\
& \nu \leq m(q-1)-1 .
\end{aligned}
$$

Proof. Let $\nu$ be an integer such that $0 \leq \nu \leq m(q-1)-1$.

We have $\theta_{0}^{0}=\varphi\left(f_{0}(z)\right)=\varphi\left(\prod_{i=1}^{q^{m}-2}\left(z-\gamma^{i}\right)\right)=\varphi\left(1+z+z^{2}+\ldots+z^{q^{m}-2}\right)=$ $\sum_{g \in G} X^{g}$.

Since $\operatorname{dim}_{\mathbb{F}_{q}}\left(C_{0}(m, q)\right)=\operatorname{dim}_{\mathbb{F}_{q}}\left(H C_{0}(m, q)\right)=q^{m}-1-\left(q^{m}-2\right)=1$, then the first assertion is proved.

For the integer $\nu$ such that $1 \leq \nu \leq m(q-1)-1, f_{\nu}(z)$ is a monic polynomial and by $(12), \operatorname{deg}\left(f_{\nu}(z)\right)=\sum_{t=1}^{m(q-1)-\nu-1} N(t)$.

Thus, $\operatorname{deg}\left(z^{j} f_{\nu}(z)\right)=j+\sum_{t=1}^{m(q-1)-\nu-1} N(t)$ for $1 \leq j \leq N(m(q-1)-\nu)-1$. By (18), for the elements $\theta_{i}^{j}$ in $K_{\nu}$, we have

$$
\operatorname{LT}\left(\theta_{\lambda}^{j}\right)<\operatorname{LT}\left(\theta_{\mu}^{k}\right) \text { if } \lambda>\mu \text {, and } \operatorname{LT}\left(\theta_{\mu}^{j}\right)<\operatorname{LT}\left(\theta_{\mu}^{k}\right) \text { if } j<k .
$$

This implies that the elements of $K_{\nu}$ are linearly independant. And by (14), they are elements of $C_{\nu}(m, q)$. 
It is clear that $\operatorname{card}\left(K_{\nu}\right)=1+\sum_{i=1}^{\nu} N(m(q-1)-i)$.

And since

$$
\begin{aligned}
N(m(q-1)-i) & =\operatorname{deg}\left(f_{i-1}(z)\right)-\operatorname{deg}\left(f_{i}(z)\right) \\
& =\left(q^{m}-1-\operatorname{deg}\left(f_{i}(z)\right)\right)-\left(q^{m}-1-\operatorname{deg}\left(f_{i-1}(z)\right)\right) \\
& =\operatorname{dim}_{\mathbb{F}_{q}}\left(H C_{i}(m, q)\right)-\operatorname{dim}_{\mathbb{F}_{q}}\left(H C_{i-1}(m, q)\right) \\
& =\operatorname{dim}_{\mathbb{F}_{q}}\left(C_{i}(m, q)\right)-\operatorname{dim}_{\mathbb{F}_{q}}\left(C_{i-1}(m, q)\right)
\end{aligned}
$$

then $\operatorname{card}\left(K_{\nu}\right)=\operatorname{dim}_{\mathbb{F}_{q}}\left(C_{\nu}(m, q)\right)$.

For $m=1$, we consider the case of the Reed-Solomon codes $C_{\nu}(1, q)$ of length $q$ and of order $\nu$ over $\mathbb{F}_{q}$.

6.2 Corollary. $C_{\nu}(1, q)$ is linearly generated over $\mathbb{F}_{q}$ by the set $\left\{\theta_{i}^{0} \mid 0 \leq i \leq \nu\right\}$ where $0 \leq \nu \leq q-2$.

\section{Example: the GRM codes of lenghth 16 over $\mathbb{F}_{4}$ in $\mathbb{F}_{4}\left[G R\left(2^{2}, 2\right)\right]$}

Let $G=\left\{0, g_{0}, g_{1}, \ldots, g_{14}\right\}$ be the additive group of the Galois ring $G R\left(2^{2}, 2\right)$ with $0<g_{0}<g_{1}<\ldots<g_{14}$.

$C_{0}(2,4)$ :

We have $C_{0}(2,4)=\varphi\left(H C_{0}(2,4)\right)$ with $H C_{0}(2,4)=\left(f_{0}(z)\right)$ and

$$
f_{0}(z)=\prod_{0<\omega_{4}(i) \leq 5}\left(z-\gamma^{i}\right)=\prod_{i=1}^{14}\left(z-\gamma^{i}\right)=1+z+z^{2}+\ldots+z^{14}
$$

where $\gamma$ is a primitive element of $\mathbb{F}_{16}$. We have $\theta_{0}^{0}=\varphi\left(f_{0}(z)\right)=X^{0}+X^{g_{0}}+$ $X^{g_{1}}+\ldots+X^{g_{14}}$. Thus, $\operatorname{LT}\left(\theta_{0}^{0}\right)=X^{g_{14}}$.

And by (15) and (16), we have

$\operatorname{dim}_{\mathbb{F}_{4}}\left(C_{0}(2,4)\right)=\operatorname{dim}_{\mathbb{F}_{4}}\left(H C_{0}(2,4)\right)=15-\operatorname{deg}\left(f_{0}(z)\right)=15-14=1$.

Thus, $C_{0}(2,4)$ is linearly generated over $\mathbb{F}_{4}$ by $\theta_{0}^{0}$.

$C_{1}(2,4)$ :

We have $C_{1}(2,4)=\varphi\left(H C_{1}(2,4)\right)$ with $H C_{1}(2,4)=\left(f_{1}(z)\right)$ and

$$
f_{1}(z)=\prod_{0<\omega_{4}(i) \leq 4}\left(z-\gamma^{i}\right) .
$$

Consider $N(5):=\operatorname{card}\left(\left\{k \in[0,15] \mid \omega_{4}(k)=5\right\}\right)=\operatorname{card}(\{11,14\})=2$.

We have $\operatorname{deg}\left(f_{1}(z)\right)=\operatorname{deg}\left(f_{0}(z)\right)-N(5)=14-2=12$.

And by (15) and (16),

$\operatorname{dim}_{\mathbb{F}_{4}}\left(C_{1}(2,4)\right)=\operatorname{dim}_{\mathbb{F}_{4}}\left(H C_{1}(2,4)\right)=15-\operatorname{deg}\left(f_{1}(z)\right)=15-12=3$. On the other hand, by (18),

since $\theta_{1}^{0}=\varphi\left(f_{1}(z)\right)$, then $\operatorname{LT}\left(\theta_{1}^{0}\right)=X^{g_{12}}$,

and since $\theta_{1}^{1}=\varphi\left(z f_{1}(z)\right)$, then $\operatorname{LT}\left(\theta_{1}^{1}\right)=X^{g_{13}}$. 
By (14), we have $C_{0}(2,4) \subset C_{1}(2,4)$. Thus, $\left\{\theta_{0}^{0}, \theta_{1}^{0}, \theta_{1}^{1}\right\}=K_{1}$ is a linearly independent set in $C_{1}(2,4)$. Then it is a linear basis of $C_{1}(2,4)$.

$C_{2}(2,4)$ :

We have $C_{2}(2,4)=\varphi\left(H C_{2}(2,4)\right)$ with $H C_{2}(2,4)=\left(f_{2}(z)\right)$ and

$$
f_{2}(z)=\prod_{0<\omega_{4}(i) \leq 3}\left(z-\gamma^{i}\right) .
$$

Consider $N(4):=\operatorname{card}\left(\left\{k \in[0,15] \mid \omega_{4}(k)=4\right\}\right)=\operatorname{card}(\{7,10,13\})=3$.

We have $\operatorname{deg}\left(f_{2}(z)\right)=\operatorname{deg}\left(f_{1}(z)\right)-N(4)=12-3=9$.

And by (15) and (16),

$\operatorname{dim}_{\mathbb{F}_{4}}\left(C_{2}(2,4)\right)=\operatorname{dim}_{\mathbb{F}_{4}}\left(H C_{2}(2,4)\right)=15-\operatorname{deg}\left(f_{2}(z)\right)=15-9=6$. On the other hand, by (18),

- since $\theta_{2}^{0}=\varphi\left(f_{2}(z)\right)$, then $\operatorname{LT}\left(\theta_{2}^{0}\right)=X^{g_{9}}$,

- since $\theta_{2}^{1}=\varphi\left(z f_{2}(z)\right)$, then $\operatorname{LT}\left(\theta_{2}^{1}\right)=X^{g_{10}}$,

- since $\theta_{2}^{2}=\varphi\left(z^{2} f_{2}(z)\right)$, then $\operatorname{LT}\left(\theta_{2}^{2}\right)=X^{g_{11}}$.

By (14), we have $C_{0}(2,4) \subset C_{1}(2,4) \subset C_{2}(2,4)$. Thus, $\left\{\theta_{0}^{0}, \theta_{1}^{0}, \theta_{1}^{1}, \theta_{2}^{0}, \theta_{2}^{1}, \theta_{2}^{2}\right\}=$ $K_{2}$ is a linearly independent set in $C_{2}(2,4)$. Then it is a linear basis of $C_{2}(2,4)$. $C_{3}(2,4)$ :

We have $C_{3}(2,4)=\varphi\left(H C_{3}(2,4)\right)$ with $H C_{3}(2,4)=\left(f_{3}(z)\right)$ and

$$
f_{3}(z)=\prod_{0<\omega_{4}(i) \leq 2}\left(z-\gamma^{i}\right) .
$$

Consider $N(3):=\operatorname{card}\left(\left\{k \in[0,15] \mid \omega_{4}(k)=3\right\}\right)=\operatorname{card}(\{3,6,9,12\})=4$.

We have $\operatorname{deg}\left(f_{3}(z)\right)=\operatorname{deg}\left(f_{2}(z)\right)-N(3)=9-4=5$.

And by (15) and (16),

$\operatorname{dim}_{\mathbb{F}_{4}}\left(C_{3}(2,4)\right)=\operatorname{dim}_{\mathbb{F}_{4}}\left(H C_{3}(2,4)\right)=15-\operatorname{deg}\left(f_{3}(z)\right)=15-5=10$. On the other hand, by (18),

- since $\theta_{3}^{0}=\varphi\left(f_{3}(z)\right)$, then $\operatorname{LT}\left(\theta_{3}^{0}\right)=X^{g_{5}}$,

- since $\theta_{3}^{1}=\varphi\left(z f_{3}(z)\right)=6$, then $\operatorname{LT}\left(\theta_{3}^{1}\right)=X^{g_{6}}$,

- since $\theta_{3}^{2}=\varphi\left(z^{2} f_{3}(z)\right)=7$, then $\operatorname{LT}\left(\theta_{3}^{2}\right)=X^{g_{7}}$,

and since $\theta_{3}^{3}=\varphi\left(z^{3} f_{3}(z)\right)=8$, then $\operatorname{LT}\left(\theta_{3}^{3}\right)=X^{g_{8}}$.

By (14), we have $C_{0}(2,4) \subset C_{1}(2,4) \subset C_{2}(2,4) \subset C_{3}(2,4)$. Thus,

$\left\{\theta_{0}^{0}, \theta_{1}^{0}, \theta_{1}^{1}, \theta_{2}^{0}, \theta_{2}^{1}, \theta_{2}^{2}, \theta_{3}^{0}, \theta_{3}^{1}, \theta_{3}^{2}, \theta_{3}^{3}\right\}=K_{3}$ is a linearly independent set in $C_{3}(2,4)$.

Then it is a linear basis of $C_{3}(2,4)$.

$C_{4}(2,4)$ :

$\overline{\text { We have }} C_{4}(2,4)=\varphi\left(H C_{4}(2,4)\right)$ with $H C_{4}(2,4)=\left(f_{4}(z)\right)$ and

$$
f_{4}(z)=\prod_{0<\omega_{4}(i) \leq 1}\left(z-\gamma^{i}\right) .
$$

Consider $N(2):=\operatorname{card}\left(\left\{k \in[0,15] \mid \omega_{4}(k)=2\right\}\right)=\operatorname{card}(\{2,5,8\})=3$.

We have $\operatorname{deg}\left(f_{4}(z)\right)=\operatorname{deg}\left(f_{3}(z)\right)-N(2)=5-3=2$.

And by (15) and (16),

$\operatorname{dim}_{\mathbb{F}_{4}}\left(C_{4}(2,4)\right)=\operatorname{dim}_{\mathbb{F}_{4}}\left(H C_{4}(2,4)\right)=15-\operatorname{deg}\left(f_{4}(z)\right)=15-2=13$. On the other hand, by (18), 
- since $\theta_{4}^{0}=\varphi\left(f_{4}(z)\right)$, then $\operatorname{LT}\left(\theta_{4}^{0}\right)=X^{g_{2}}$,

- since $\theta_{4}^{1}=\varphi\left(z f_{4}(z)\right)=3$, then $\operatorname{LT}\left(\theta_{4}^{1}\right)=X^{g_{3}}$,

- since $\theta_{4}^{2}=\varphi\left(z^{2} f_{4}(z)\right)=4$, then $\operatorname{LT}\left(\theta_{4}^{2}\right)=X^{g_{4}}$.

By (14), we have $C_{0}(2,4) \subset C_{1}(2,4) \subset C_{2}(2,4) \subset C_{3}(2,4) \subset C_{4}(2,4)$. Thus,

$\left\{\theta_{0}^{0}, \theta_{1}^{0}, \theta_{1}^{1}, \theta_{2}^{0}, \theta_{2}^{1}, \theta_{2}^{2}, \theta_{3}^{0}, \theta_{3}^{1}, \theta_{3}^{2}, \theta_{3}^{3}, \theta_{4}^{0}, \theta_{4}^{1}, \theta_{4}^{2}\right\}=K_{4}$ is a linearly independent set in $C_{4}(2,4)$. Then it is a linear basis of $C_{4}(2,4)$.

$C_{5}(2,4)$ :

$\overline{\text { We have }} C_{5}(2,4)=\varphi\left(H C_{5}(2,4)\right)$ with $H C_{5}(2,4)=\left(f_{5}(z)\right)$ and

$$
f_{5}(z)=\prod_{0<\omega_{4}(i) \leq 0}\left(z-\gamma^{i}\right)=1 .
$$

Then $\operatorname{deg}\left(f_{5}(z)\right)=0$.

And by (15) and (16),

$\operatorname{dim}_{\mathbb{F}_{4}}\left(C_{5}(2,4)\right)=\operatorname{dim}_{\mathbb{F}_{4}}\left(H C_{5}(2,4)\right)=15-\operatorname{deg}\left(f_{5}(z)\right)=15$. On the other hand, by (18),

- since $\theta_{5}^{0}=\varphi\left(f_{5}(z)\right)$, then $\operatorname{LT}\left(\theta_{5}^{0}\right)=X^{g_{0}}$,

- since $\theta_{5}^{1}=\varphi\left(z f_{5}(z)\right)=1$, then $\operatorname{LT}\left(\theta_{5}^{1}\right)=X^{g_{1}}$.

By (14), we have $C_{0}(2,4) \subset C_{1}(2,4) \subset C_{2}(2,4) \subset C_{3}(2,4) \subset C_{4}(2,4) \subset$ $C_{5}(2,4)$. Thus,

$\left\{\theta_{0}^{0}, \theta_{1}^{0}, \theta_{1}^{1}, \theta_{2}^{0}, \theta_{2}^{1}, \theta_{2}^{2}, \theta_{3}^{0}, \theta_{3}^{1}, \theta_{3}^{2}, \theta_{3}^{3}, \theta_{4}^{0}, \theta_{4}^{1}, \theta_{4}^{2}, \theta_{5}^{0}, \theta_{5}^{1}\right\}=K_{5}$ is a linearly independent set in $C_{5}(2,4)$. Then it is a linear basis of $C_{5}(2,4)$.

\section{References}

[1] H. Andriatahiny, The Generalized Reed-Muller codes and the Radical Powers of a modular algebra, British Journal of Mathematics and Computer Science, 18(5):1-14, 2016.

[2] E. F. Assmus and J. D. Key, Polynomial codes and finite geometries, Handbook of Coding Theory, 1994.

[3] S. D. Berman, On the theory of group codes, Kibernetika 3(1) (1967), pp.3139.

[4] P. Charpin, Une généralisation de la construction de Berman des codes de Reed et Muller p-aires, Communications in algebra 16 (1988), pp.22312246 .

[5] Couselo E., Gonzalez S., Markov VT., Martinez C., Nechaev AA., Ideal representation of Reed-Solomon and Reed-Muller codes, Algebra and Logic, vol.51, no.3 (2012).

[6] D. Cox, J. Little and D. O'Shea, Ideals, Varieties and Algorithms, Springer, 1992.

[7] S.A. Jennings, The structure of the group ring of a p-group over a modular field, Trans.Amer.Math.Soc., 50:175-185, 1941. 
[8] T. Kasami, S. Lin and W. W. Peterson, New generalizations of the ReedMuller codes, IEEE Transactions on Information Theory 14 (1968), no.2, pp.189-205.

[9] Landrock P. and Manz O., Classical Codes as Ideals in Group Algebras, Designs, Codes and Cryptography, 2 (1992), pp.273-285.

[10] B.R. McDonald, Finite Rings with identity, Dekker, 1974.

[11] J.H. Van Lint, Introduction to Coding Theory, 3rd ed., Springer, 1998. 